\title{
Modified Block BiCGSTAB for Lattice QCD
}

\author{
Y. Nakamura ${ }^{* a}$, K. -I. Ishikawa ${ }^{b}$, Y. Kuramashi ${ }^{c, d, a, f}$, T. Sakurai ${ }^{e, f}$, H. Tadano ${ }^{e, d, f}$ \\ ${ }^{a}$ RIKEN Advanced Institute for Computational Science, Kobe, Hyogo 650-0047, Japan \\ ${ }^{b}$ Graduate School of Science, Hiroshima University, Higashi-Hiroshima, Hiroshima 739-8526, \\ Japan \\ ${ }^{c}$ Graduate School of Pure and Applied Sciences, University of Tsukuba, Tsukuba, Ibaraki \\ 305-8571, Japan \\ ${ }^{d}$ Center for Computational Sciences, University of Tsukuba, Tsukuba, Ibaraki 305-8577, Japan \\ ${ }^{e}$ Department of Computer Science, University of Tsukuba, Tsukuba, Ibaraki 305-8573, Japan \\ ${ }^{f}$ JST, CREST, 5, Sanbancho, Chiyoda-ku, Tokyo 102-0075, Japan \\ E-mail: nakamura@riken.jp
}

We presented "Modified block BiCGSTAB for lattice QCD", results for application of block BiCGSTAB algorithm modified by the QR decomposition and the SAP preconditioner to the Wilson-Dirac equation with multiple right-hand sides in lattice QCD on $32^{3} \times 64$ and $64^{4}$ lattices at almost physical quark masses.

\section{Reference:}

Y. Nakamura, K.-I. Ishikawa, Y. Kuramashi, T. Sakurai, and H. Tadano,

Modified block BiCGSTAB for lattice QCD, Comput. Phys. Commun. 183 (2012) 34.

The XXIX International Symposium on Lattice Field Theory

July 10-16, 2011

Squaw Valley, California, USA

\footnotetext{
* Speaker.
} 